\title{
Homocysteine is associated with plasma high-sensitivity cardiac troponin T levels in a community-dwelling population
}

This article was published in the following Dove Press journal:

Clinical Interventions in Aging

27 December 2013

Number of times this article has been viewed

\section{Ruihua Cao* \\ Yongyi Bai* \\ Ruyi Xu \\ Ping Ye}

Department of Geriatric Cardiology,

Chinese PLA General Hospital,

Beijing, People's Republic of China

*These authors contributed equally to this work
Correspondence: Ping Ye

Department of Geriatric Cardiology,

Chinese PLA General Hospital,

No 28, Fuxing Road, Beijing 100853 ,

People's Republic of China

Tel +86 I0 66876369

Fax +861066876349

Email yeping30I@sina.com
Objectives: Homocysteine (HCY) is associated with an increased risk for cardiovascular disease, possibly leading to myocardial damage. Cardiac troponin $\mathrm{T}$ (TnT), a marker of cardiomyocyte injury, can be detected by high-sensitivity TnT (hsTnT) assay. The current study investigated the relationship between plasma HCY and hsTnT levels in a communitybased population.

Methods: We related plasma levels of hsTnT to HCY levels in 1,497 participants (mean age, 62.4 years; 629 men, 868 women) from a community-based population in Beijing, People's Republic of China.

Results: In multiple logistic regression models, serum HCY was associated with a higher likelihood of detectable hsTnT (odds ratio $1.5 ; 95 \%$ confidence interval $1.07-2.10 ; P=0.018$ ). A subsequent subgroup analysis found that in subjects aged 65 years and older, the association between hsTnT levels and HCY levels was strengthened. The association between hsTnT and $\mathrm{HCY}$ was not present in the younger subgroup ( $<65$ years old).

Conclusion: Levels of serum HCY are associated with hsTnT levels in the elderly, indicating a relationship between HCY and subclinical myocardial damage.

Keywords: epidemiology, myocardial damage, markers, relationship, subclinical

\section{Introduction}

Homocysteine (HCY) is a sulfur-containing amino acid formed during the metabolism of methionine. HCY has been reported to be a critical predictor of cardiovascular events, particularly in older individuals. ${ }^{1}$ Accumulating data from epidemiological studies suggests that individuals with an elevated blood level of total HCY have increased risk of stroke and cardiovascular disease. The list of well-known cardiovascular risk factors, like oxidative stress, inflammation, chronic hyperglycemia, and hypercholesterolemia, is becoming expanded by $\mathrm{HCY}^{2}$ Whether levels of HCY on the cardiovascular system are associated with myocardial damage is not clear.

Cardiac troponin $\mathrm{T}$ ( $\mathrm{TnT}$ ), a 37-kD polypeptide expressed by cardiac myocytes, is released in the setting of myocardial injury. Minimally elevated levels of TnT have also been found in small subsets of the general population; the cause and significance of these rare elevations are unclear, but the elevations seem to indicate a worse prognosis. ${ }^{3,4}$ Recent advances have resulted in the development of high sensitivity troponin assays that meet the recommendations established by the International Federation of Clinical Chemistry and Laboratory Medicine. ${ }^{5}$ Several studies have reported enhanced diagnostic and prognostic values of these high-sensitivity cardiac troponin $\mathrm{T}$ (hsTnT) assays across a spectrum of cardiovascular disease, including 
acute myocardial infarction, ${ }^{6,7}$ stable coronary heart disease with preserved left ventricular function, ${ }^{8}$ and heart failure. ${ }^{9}$ In addition, circulating hsTnT is detectable and has a prognostic value in a community-dwelling population, ${ }^{10}$ raising the possibility of the presence of subclinical silent myocardial damage in subjects without overt cardiovascular disease.

Whether HCY is associated with the release of very low levels of cardiac TnT in asymptomatic subjects has not been well established yet. The present study investigated the association between these two biomarkers in a communitydwelling population without overt cardiovascular disease.

\section{Method}

\section{Study population and design}

This was a community-based cross-sectional study of people living in the Pingguoyuan area of the Shijingshan district, a metropolitan area of Beijing, People's Republic of China. After a routine health checkup between September 2007 and January 2009, initially, a total of 1,859 permanent residents were recruited to the study as described previously. ${ }^{11}$ Thirtyone subjects with bedridden status, mental illness, and severe systemic diseases were excluded from the analysis.

Clinical data collection and biomarker variable determination were performed in 1,828 subjects. Adequate measurements were either not attempted or not obtained in 86 participants. A total of 141 participants with overt cardiovascular disease (defined as a composite of coronary heart disease [recognized or unrecognized myocardial infarction, angina pectoris, or coronary insufficiency], cerebrovascular disease [stroke or transient ischemic attack], congestive heart failure, or intermittent claudication) were excluded. Thus, cardiac biomarkers were tested in 1,601 subjects. Among them, a total of 1,497 subjects (mean age 62.4 years; range 45-96 years) with available hsTnT results were eligible for analysis. The study was approved by the ethics committee of the Chinese People's Liberation Army (PLA) General Hospital, and each participant provided written informed consent.

\section{Clinical data collection}

Information regarding medical history, family history of cardiovascular disease, and lifestyle were obtained through self-reporting standardized questionnaires. Anthropometrics were measured in the clinic, with participants wearing light clothing and no shoes. Height was measured in centimeters using a wall-mounted measuring tape, and weight was measured in kilograms using a digital scale. The resting heart rate was measured by cardiac auscultation for 1 minute.
We defined current smokers as individuals who smoked any tobacco in the previous 12 months and included those who had quit within the past year.

Systolic and diastolic blood pressures (SBP and DBP) were measured by well trained staff members on the right arm of the seated participant, after at least 5 minutes of rest, with a mercury sphygmomanometer and an appropriately sized cuff. Three sequential blood pressure measurements were obtained, and the average was used for analyses.

\section{Biomarker variable determination}

All subjects underwent full laboratory evaluation (lipid profile, and liver and kidney function indices). Blood samples were obtained between 8 am and 10 am from fasting participants after they had been in the supine position for 10-15 minutes. Samples were centrifuged immediately and stored at $-80^{\circ} \mathrm{C}$ until assays were performed. Concentrations of HCY were assayed by high-performance chromatography with fluorometric detection. Concentrations of fasting glucose, total cholesterol (TC), triglyceride (TG), high-density lipoprotein cholesterol (HDL-C), low-density lipoprotein cholesterol (LDL-C), and uric acid were determined using the Roche enzymatic assays (Roche Diagnostics GmbH, Mannheim, Germany) on a Roche autoanalyzer (Roche Diagnostics, Indianapolis, IN, USA). Concentrations of highsensitivity C-reactive protein were determined by immunoturbidimetric assay (Siemens Healthcare Diagnostics, IN, USA) using a Dimension RxL Max analyzer (Siemens Healthcare Diagnostics). Concentrations of serum creatinine were measured by enzymatic assay (Roche Diagnostics $\mathrm{GmbH}$ ) on a Hitachi 7600 autoanalyzer (Hitachi, Tokyo, Japan). Concentrations of hsTnT were determined using a precommercial hsTnT assay (Roche Diagnostics $\mathrm{GmbH}$ ) on a Modular Analytics E170 autoanalyzer (Roche Diagnostics). Given enhanced sensitivity, this assay was reported in units of pictograms per milliliter, with an interassay coefficient of variation of $8 \%$ at $10 \mathrm{pg} / \mathrm{mL}$ and $2.5 \%$ at $100 \mathrm{pg} / \mathrm{mL}^{8}$ The low detection limit of the hsTnT assay was $3.0 \mathrm{pg} / \mathrm{mL}$ (according to manufacturer's information).

All testing was performed by well trained personnel blinded to clinical data, in the Department of Biochemistry of Chinese PLA General Hospital.

\section{Definition of variables}

Body mass index (BMI) was calculated as weight in kilograms divided by height in meters squared. Hypertension was defined as a mean SBP $\geq 140 \mathrm{mmHg}$, mean DBP $\geq 90 \mathrm{mmHg}$, or use of antihypertensive medication. Diabetes mellitus 
was defined as a fasting glucose $\geq 7.0 \mathrm{mmol} / \mathrm{L}$, nonfasting glucose $\geq 11.1 \mathrm{mmol} / \mathrm{L}$, or use of antihyperglycemic medication. ${ }^{12}$ Hyperhomocysteinemia was defined as HCY levels $\geq 15 \mu \mathrm{mol} / \mathrm{L}^{13}$

\section{Statistical analyses}

Continuous variables are presented as mean \pm standard deviation (SD) or median (with interquartile range); dichotomous variables are presented as numbers and percentages. Levels of HCY and hsTnT were natural logarithmically (Ln) transformed to normalize their distributions. The hsTnT was classified as detectable ( $\geq 3 \mathrm{pg} / \mathrm{mL}$ ) or undetectable. The $\mathrm{HCY}$ was classified as high level group ( $\geq 15 \mu \mathrm{mol} / \mathrm{L})$ or normal level group $(<15 \mu \mathrm{mol} / \mathrm{L})$. Differences in LnhsTnT levels between high and normal HCY groups were analyzed with Student's $t$-tests.

We assessed the associations of HCY levels with manifestations of subclinical myocardial damage (detectable hsTnT versus undetectable) by means of logistic regression models, hsTnT was treated as the dependent variable (detectable hsTnT versus undetectable). Forward stepwise multiple logistic regression analysis was performed to obtain the odds ratios (ORs) and 95\% confidence intervals (CIs). Regression models were adjusted for age and sex as the independent variable (Model 1) and additionally adjusted for hypertension, diabetes, SBP, DBP, current smoking, BMI, heart rate, levels of plasma fasting glucose, TC, TG, and HDL-C as the independent variable (Model 2).

Because age is an important determinant of HCY levels, we repeated the regression analyses for participants younger $(n=851)$ and older $(n=646)$ than 65 years of age.

All statistical analyses were performed using Stata software (Stata Corporation, College Station, TX, USA), version 9.0. A two-sided value of $P<0.05$ was considered significant.

\section{Results \\ Characteristics}

Altogether, we included 1,497 participants in the present study. The mean ( \pm SD) age of participants in the study was $62.36 \pm 9.81$ years, and $58.0 \%$ were women. The characteristics of the study population are summarized in Table 1.

\section{Distribution of hs TnT concentrations}

Among the 1,497 participants, 807 (53.9\%) had detectable values of hsTnT ( $\geq 3.0 \mathrm{pg} / \mathrm{mL})$. The range of detectable hsTnT concentrations was 3.03-176.40 pg/mL, with a median value of $7.45 \mathrm{pg} / \mathrm{mL}$ (quartile 1 to quartile 3 : 4.84-12.02 pg/mL).

\section{$\mathrm{HCY}$ and the presence of detectable hsTnT}

The levels of hsTnT in the high HCY group were higher than that in the normal HCY group $(P<0.001)$ (Figure 1).

Table I Characteristics of the study sample

\begin{tabular}{|c|c|c|c|c|}
\hline Variable & $\begin{array}{l}\text { All subjects } \\
(n=I, 497)\end{array}$ & $\begin{array}{l}\mathrm{HCY}<15 \mu \mathrm{mol} / \mathrm{L} \\
(\mathrm{n}=468)\end{array}$ & $\begin{array}{l}\mathrm{HCY} \geq 15 \mu \mathrm{mol} / \mathrm{L} \\
(\mathrm{n}=1,029)\end{array}$ & $P$-value \\
\hline Age, years & $62.36 \pm 9.81$ & $57.23 \pm 8.57$ & $64.69 \pm 9.46$ & $<0.001$ \\
\hline $\mathrm{BMI}, \mathrm{kg} / \mathrm{m}^{2}$ & $25.6 I \pm 3.5 I$ & $25.28 \pm 3.48$ & $25.76 \pm 3.52$ & 0.013 \\
\hline Waist circumference, $\mathrm{cm}$ & $86.90 \pm 9.79$ & $84.05 \pm 9.65$ & $88.19 \pm 9.57$ & $<0.001$ \\
\hline $\mathrm{SBP}, \mathrm{mmHg}$ & $130.79 \pm 18.20$ & $127.29 \pm 17.48$ & $|32.38 \pm| 8.3 \mid$ & $<0.001$ \\
\hline $\mathrm{DBP}, \mathrm{mmHg}$ & $76.86 \pm|0.7|$ & $76.37 \pm 9.94$ & $77.09 \pm 11.04$ & 0.231 \\
\hline Heart rate, beats/min & $75.28 \pm 9.95$ & $75.12 \pm 8.95$ & $75.35 \pm 10.38$ & 0.675 \\
\hline $\mathrm{TC}, \mathrm{mmol} / \mathrm{L}$ & $5.07 \pm 0.91$ & $5.12 \pm 0.94$ & $5.05 \pm 0.90$ & 0.184 \\
\hline TG, mmol/L & $1.81 \pm 1.22$ & $1.76 \pm 1.11$ & $1.84 \pm 1.27$ & 0.258 \\
\hline HDL-C, mmol/L & $I .4 I \pm 0.36$ & $1.46 \pm 0.40$ & $1.38 \pm 0.34$ & $<0.001$ \\
\hline LDL-C, mmol/L & $2.99 \pm 0.70$ & $2.97 \pm 0.72$ & $2.99 \pm 0.70$ & 0.506 \\
\hline Fasting glucose, $\mathrm{mmol} / \mathrm{L}$ & $5.42 \pm 1.68$ & $5.62 \pm 1.89$ & $5.34 \pm 1.57$ & 0.003 \\
\hline $\begin{array}{l}\text { I20-minute postprandial } \\
\text { glucose, } \mathrm{mmol} / \mathrm{L}\end{array}$ & $7.99 \pm 4.23$ & $7.91 \pm 4.17$ & $8.02 \pm 4.26$ & 0.668 \\
\hline Detectable hsTnT, n (\%) & $816(54.5)$ & 201 (42.9) & $615(59.8)$ & $<0.001$ \\
\hline Current smoking, n (\%) & $384(25.7)$ & $75(16.0)$ & $309(30.0)$ & $<0.001$ \\
\hline Diabetes, n (\%) & $262(17.5)$ & $88(18.8)$ & $174(16.9)$ & 0.371 \\
\hline Hypertension, n (\%) & $663(44.3)$ & $164(35.0)$ & $499(48.5)$ & $<0.001$ \\
\hline
\end{tabular}

Notes: BMI calculated using height, weight, waist circumference; TC, HDL-C, and LDL-C are given as mean \pm standard deviation; TG and HCY values given as median (quartile I, quartile 3).

Abbreviations: BMI, body mass index; DBP, diastolic blood pressure; HCY, homocysteine; HDL-C, high-density lipoprotein cholesterol; hsTnT, high-sensitivity troponin T; LDL-C, low-density lipoprotein cholesterol; SBP, systolic blood pressure; TC, total plasma cholesterol; TG, triglycerides. 


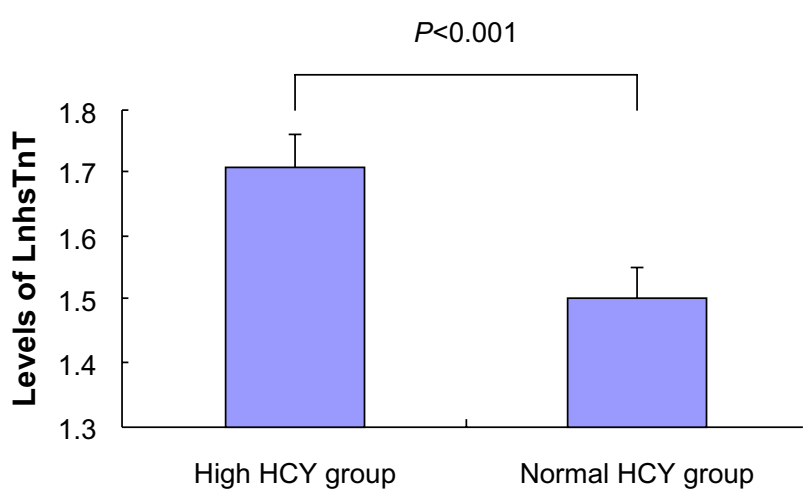

Figure I Levels of hsTnT were Ln transformed to normalize their distributions. Plasma levels of LnhsTnT in high and normal HCY group. The levels of LnhsTnT in the high HCY group were higher than that in the normal HCY group $(P<0.00 \mathrm{I})$.

Abbreviations: HCY, homocysteine; hsTnT, high-sensitivity TnT; Ln, natural logarithmically.

On univariate logistic regression analysis, the presence of detectable and undetectable hsTnT was significantly related to HCY level $(P<0.001)$. In the adjusted models ( 1 and 2$)$, the relationship remained statistically significant (Table 2 ). Model 2 showed that, after adjusting for age, sex, hypertension, diabetes, SBP, DBP, current smoking, BMI, heart rate, levels of plasma fasting glucose, TC, TG, and HDL-C, every SD increase in LnHCY levels was associated with a 1.5-higher likelihood of the presence of detectable hsTnT (OR [per SD increase in LnHCY] 1.5; 95\% CI 1.07-2.10; $P=0.018)$.

Subsequently, a subgroup analysis according to participant age was conducted. In the group older than 65 years, the association between HCY levels (OR 1.87, 95\% CI 1.05-3.36, $P=0.035$ ) and the presence of detectable hsTnT was strengthened. However, in participants younger than 65 years, the relationship between HCY levels (OR 1.22,

Table 2 Relationship between levels of $\mathrm{HCY}$ and levels of hsTnT

\begin{tabular}{lll}
\hline & \multicolumn{2}{l}{ Levels of LnHCY } \\
\cline { 2 - 3 } & OR (95\% Cl) & P-value \\
\hline $\begin{array}{lll}\text { Detectable hsTnT } \\
\text { Unadjusted }\end{array}$ & $3.06(2.28-4.09)$ & $<0.00 \mathrm{I}$ \\
Model I & $\mathrm{I} .4 \mathrm{I}(\mathrm{I} .02-1.95)$ & 0.035 \\
Model 2 & $\mathrm{I} .5(1.07-2.10)$ & 0.018 \\
\hline
\end{tabular}

Notes: Levels of HCY were Ln transformed to normalize their distributions. Data are presented as ORs (per SD increase in LnHCY levels) and corresponding 95\% $\mathrm{Cls}$. In the logistic regression model, detectable hsTnT was treated as the dependent variable (detectable hsTnT versus undetectable). Model I is adjusted for age and sex as the independent variables. Model 2 is additionally adjusted for the following independent variables: hypertension, diabetes, SBP, DBP, current smoking, BMI, heart rate, levels of plasma fasting glucose, TC, TG, and HDL-C.

Abbreviations: $\mathrm{BMI}$, body mass index; $\mathrm{Cl}$, confidence interval; $\mathrm{DBP}$, diastolic blood pressure; HCY, homocysteine; HDL-C, high-density lipoprotein cholesterol; hsTnT, high-sensitivity TnT; Ln, natural logarithmically; OR, odds ratio; SBP, systolic blood pressure; SD, standard deviation; TC, total plasma cholesterol; TG, triglycerides; $\mathrm{TnT}$, troponin $\mathrm{T}$.
95\% CI $0.8-1.87, P=0.356)$ and detectable hsTnT disappeared (Table 3).

\section{Discussion}

In this study, we demonstrated for the first time that, in a community-dwelling asymptomatic population, levels of HCY were positively associated with the presence of detectable hsTnT, independent of age, sex, or other vascular risk factors. These results indicated an independent association between $\mathrm{HCY}$ and subclinical myocardial injury. Notably, in the predefined subgroup analysis, the association between levels of HCY and subclinical myocardial injury was strengthened in elderly participants, but not present in those younger than 65 years old.

The list of well-known cardiovascular risk factors, such as oxidative stress, inflammation, chronic hyperglycemia, and hypercholesterolemia, is becoming expanded by new candidates, including the elevated concentrations of some excitotoxic amino acids, such as HCY. Previous studies have investigated associations of HCY with different indices of arterial stiffness. ${ }^{14,15}$ In the present study, we demonstrated an association between levels of HCY and increased presence of detectable hsTnT, which is a sensitive marker for the assessment of myocardial damage. The findings of the present study suggest that the higher levels of HCY might be partly mediated myocardial damage.

There are several ways that HCY predisposes cardiac damage, including direct damage, damage of endothelial function, and lipid metabolism disorder. First, HCY induces cardiomyocyte dysfunction and apoptosis through increase in oxidant stress. ${ }^{16}$ Second, one possible mechanism to explain

Table 3 Relationship between levels of HCY and levels of hsTnT for participants younger and older than the age of 65 years

\begin{tabular}{|c|c|c|}
\hline & \multicolumn{2}{|c|}{ Levels of LnHCY } \\
\hline & OR (95\% Cl) & $P$-value \\
\hline \multicolumn{3}{|l|}{ Detectable hsTnT } \\
\hline All subjects $(n=I, 497)$ & I.5 (I.07-2.10) & 0.018 \\
\hline \multicolumn{3}{|l|}{ Age, years } \\
\hline$\geq 65(n=646)$ & $1.87(1.05-3.36)$ & 0.035 \\
\hline$<65(n=85 I)$ & I.22 (0.8-I.87) & 0.356 \\
\hline
\end{tabular}

Notes: Levels of $\mathrm{HCY}$ were natural logarithmically $(\mathrm{Ln})$ transformed to normalize their distributions. Data are presented as ORs (per SD increase in LnHCY levels) and corresponding $95 \% \mathrm{Cls}$. In the logistic regression model (Model 2), detectable hsTnT was treated as the dependent variable (detectable hsTnT versus undetectable); age, sex, hypertension, diabetes, SBP, DBP, current smoking, BMI, heart rate, levels of plasma fasting glucose, TC, TG, and HDL-C are treated as independent variables. Abbreviations: $\mathrm{BMI}$, body mass index; $\mathrm{Cl}$, confidence interval; $\mathrm{DBP}$, diastolic blood pressure; HCY, homocysteine; HDL-C, high-density lipoprotein cholesterol; hsTnT, high-sensitivity TnT; Ln, natural logarithmically; OR, odds ratio; SBP, systolic blood pressure; SD, standard deviation; TC, total plasma cholesterol; TG, triglycerides; $\mathrm{TnT}$, troponin $\mathrm{T}$. 
the effect of HCY in decreasing cardiomyocyte contractility is that the effect is mediated by the reduced responsiveness of myofilaments to $\mathrm{Ca}^{2+}$. The interaction between myofilaments and $\mathrm{Ca}^{2+}$ is suggested to be regulated by the phosphorylation status of myofilament proteins. ${ }^{17}$ Third, HCY has been shown to promote endothelial dysfunction by decreasing the bioavailability of nitric oxide and increasing oxidative stress in the vasculature. ${ }^{16}$ Hyperhomocysteinemia has been shown to severely impair cardiac relaxation and contractile function. The mechanism may include hyperhomocysteinemia-induced functional perturbation of endothelial cells, vascular smooth muscle cells, and platelets, as well as effects on components of the coagulation and/or fibrinolytic systems. ${ }^{18}$ Finally, it is possible that the inhibition of apoA-I synthesis, improvement of acetylated-LDL uptake, induced by HCY, is a subsequent mechanism through which HCY is linked with lipid metabolism and atherosclerosis development. ${ }^{19}$ Thus, the relationship between HCY and observed hsTnT may merely imply the association between the underlying subclinical coronary atherosclerosis and heart failure with minimal myocardial damage. The supplementation of folic acid can be used as an adjuvant therapy in cardiovascular alterations caused by homocysteine. ${ }^{20}$

In this study, HCY were associated with subclinical myocardial damage only in elderly participants. This difference can be attributed to a number of factors. First, our study population is relatively old, with $43.2 \%$ of the participants being $\geq 65$ years of age (40-90; median 62 ). Although HCY increases with age, the steepest rise occurs after the age of 65 years, resulting in strengthened subclinical myocardial damage in older subjects. Second, age itself is among the most important cardiovascular risk factors, heavily modifying the strength of others. Third, elderly subjects had a higher presence of other vascular risk factors than younger participants, these factors can interact with HCY, which may increase the susceptibility to the influence of artery stiffening in this group of subjects. ${ }^{21}$

The present study has limitations. First, the present study was performed in Chinese residents from one community in Beijing; the results may not represent Chinese from other areas of the People's Republic of China, and might not be applicable to other ethnic groups. Second, because of the cross-sectional design and its inherent limitations, the present study cannot determine causal relationships between the associations. Accordingly, our observations need confirmation in longitudinal and interventional studies. Third, a significant proportion of residents were excluded because of missing or inadequate clinical and biomarker data.
This is a well-known but unavoidable limitation of large epidemiological studies that may bias toward the null hypothesis because of loss of cases that presumably had more extreme values for the analyzed variables. Fourth, though the results are adjusted for multiple covariates that may be associated with hsTnT levels or with altered vascular properties, the possibility of residual confounding remains.

There are several strengths to our study. First, to the best of our knowledge, the present investigation is the first study evaluating the association between the levels of HCY and subclinical myocardial damage in a large community-based population. Second, we included the most sensitive detection method of myocardial damage (hsTnT), which enabled us to explore the relationship between plasma levels of HCY and subclinical myocardial injury.

\section{Conclusion}

In conclusion, we observed robust, cross-sectional association between the levels of HCY and hsTnT, suggesting a consistent positive relationship between plasma HCY levels and subclinical myocardial damage, particularly in the elderly subjects. Considering the cross-sectional design of the present study, long-term follow-up studies are needed to evaluate whether HCY causes such manifestations of subclinical myocardial injury.

\section{Acknowledgments}

This work was supported by grants from the Key National Basic Research Program of China (2013CB530804) and Nature Science Foundation of China (81270941) to P Ye, and the Nature Science Foundation of China (81100878) and the Beijing Nova Program (Z121107002513124) to Y Bai.

\section{Disclosure}

The authors have no conflicts of interest to declare.

\section{References}

1. Homocysteine Studies Collaboration. Homocysteine and risk of ischemic heart disease and stroke: a meta-analysis. JAMA. 2002;288(16): 2015-2022.

2. Karolczak K, Kamysz W, Karafova A, Drzewoski J, Watala C. Homocysteine is a novel risk factor for suboptimal response of blood platelets to acetylsalicylic acid in coronary artery disease: a randomized multicenter study. Pharmacol Res. 2013;74:7-22.

3. Wallace TW, Abdullah SM, Drazner MH, et al. Prevalence and determinants of troponin $\mathrm{T}$ elevation in the general population. Circulation. 2006;113(16):1958-1965.

4. Zethelius B, Johnston N, Venge P. Troponin I as a predictor of coronary heart disease and mortality in 70-year-old men: a community-based cohort study. Circulation. 2006;113(8):1071-1078. 
5. Apple FS, Jesse RL, Newby LK, et al. National Academy of Clinical Biochemistry and IFCC Committee for Standardization of Markers of Cardiac Damage Laboratory Medicine Practice Guidelines: analytical issues for biochemical markers of acute coronary syndromes. Circulation. 2007;115(13):e352-e355.

6. Reichlin T, Hochholzer W, Bassetti S, et al. Early diagnosis of myocardial infarction with sensitive cardiac troponin assays. $N$ Engl J Med. 2009;361(9):858-867.

7. Keller T, Zeller T, Peetz D, et al. Sensitive troponin I assay in early diagnosis of acute myocardial infarction. $N$ Engl J Med. 2009;361(9): $868-877$.

8. Omland T, de Lemos JA, Sabatine MS, et al. A sensitive cardiac troponin T assay in stable coronary artery disease. $N$ Engl J Med. 2009;361(26):2538-2547.

9. Latini R, Masson S, Anand IS, et al. Prognostic value of very low plasma concentrations of troponin $\mathrm{T}$ in patients with stable chronic heart failure. Circulation. 2007;116(11):1242-1249.

10. Otsuka T, Kawada T, Ibuki C, Seino Y. Association between highsensitivity cardiac troponin $\mathrm{T}$ levels and the predicted cardiovascular risk in middle-aged men without overt cardiovascular disease. Am Heart J. 2010;159(6):972-978.

11. Bai Y, Ye P, Luo L, et al. Arterial stiffness is associated with minimally elevated high-sensitivity cardiac, troponin T levels in a communitydwelling population. Atherosclerosis. 2011;218(2):493-498.

12. Alberti KG, Eckel RH, Grundy SM, et al. Harmonizing the metabolic syndrome: a Joint Interim Statement of the International Diabetes Federation Task Force on Epidemiology and Prevention; National Heart, Lung, and Blood Institute; American Heart Association; World Heart Federation; International Atherosclerosis Society; and International Association for the Study of Obesity. Circulation. 2009;120(16):1640-1645.
13. Rossi GP, Maiolino G, Seccia TM, et al. Hyperhomocysteinemia predicts total and cardiovascular mortality in high-risk women. J Hypertens. 2006;24(5):851-859.

14. Nestel PJ, Chronopoulos A, Cehun M. Arterial stiffness is rapidly induced by raising the plasma homocysteine concentration with methionine. Atherosclerosis. 2003;171(1):83-86.

15. Anan F, Masaki T, Umeno Y et al. Correlations between homocysteine levels and atherosclerosis in Japanese type 2 diabetic patients. Metabolism. 2007;56(10):1390-1395.

16. Wang X, Cui L, Joseph J, et al. Homocysteine induces cardiomyocyte dysfunction and apoptosis through p38MAPK-mediated increase in oxidant stress. J Mol Cell Cardiol. 2012;52(3):753-760.

17. Liao P, Wang SQ, Wang S, et al. p38 mitogen-activated protein kinase mediates a negative inotropic effect in cardiac myocytes. Circ Res. 2002;90(2):190-196.

18. Durand P, Prost M, Loreau N, Lussier-Cacan S, Blache D. Impaired homocysteine metabolism and atherothrombotic disease. Lab Invest. 2001;81(5):645-672.

19. Mierzecki A, Ktoda K, Bukowska H, Chetstowski K, MakarewiczWujec M, Koztowska-Wojciechowska M. Association between low-dose folic acid supplementation and blood lipids concentrations in male and female subjects with atherosclerosis risk factors. Med Sci Monit. 2013;19:733-739.

20. Kolling J, Scherer EB, da Cunha AA, da Cunha MJ, Wyse AT. Homocysteine induces oxidative-nitrative stress in heart of rats: prevention by folic acid. Cardiovasc Toxicol. 2011;11(1):67-73.

21. Cameron JD, Bulpitt CJ, Pinto ES, Rajkumar C. The aging of elastic and muscular arteries: a comparison of diabetic and nondiabetic subjects. Diabetes Care. 2003;26(7):2133-2138.
Clinical Interventions in Aging

\section{Publish your work in this journal}

Clinical Interventions in Aging is an international, peer-reviewed journal focusing on evidence-based reports on the value or lack thereof of treatments intended to prevent or delay the onset of maladaptive correlates of aging in human beings. This journal is indexed on PubMed Central, MedLine, the American Chemical Society's 'Chemical Abstracts

\section{Dovepress}

Service' (CAS), Scopus and the Elsevier Bibliographic databases. The manuscript management system is completely online and includes a very quick and fair peer-review system, which is all easy to use. Visit $\mathrm{http}: / /$ www.dovepress.com/testimonials.php to read real quotes from published authors. 